\title{
On a Unified Theory of Some Nonhomogeneous Poisson Process Models for Software Reliability
}

\author{
Rong-Huei Hou \\ Sy-Yen Kuo
Department of Electrical Engineering
National Taiwan University
Taipei, Taiwan
Email: sykuo@cc.ee.ntu.edu.tw

Yi-Ping Chang ${ }^{1}$

\begin{abstract}
In this paper, we show how several existing software reliability growth models based on nonhomogeneous Poisson processes (NHPPs) can be comprehensively derived by applying the concept of the weighted arithmetic, weighted geometric, or weighted harmonic mean. Furthermore, based on these three weighted means, we thus proposed a more general NHPP model from the viewpoint of quasi-arithmetic mean. Under this general framework, we can not only derive some existing NHPP models but also can derive some new NHPP models.
\end{abstract}

\section{Introduction}

Computer systems have been widely applied in the control of many complex and critical systems, such as nuclear reactors, space shuttles, chemical plants, etc. The breakdown of a computer system, caused by software errors, may result in tremendous damage for social life. Therefore, it is very important to develop a highly reliable software system, and software reliability is one of the key issues in the software product development. Models, which are concerned with the relationship between the time span of testing a program and the cumulative number of errors discovered, are called software reliability growth models (SRGMs) [1]. Many software reliability growth models have been proposed and investigated [2-7].

Model unification is very useful for the study of general models without making many assumptions. There are some model unification schemes in the literature

\footnotetext{
${ }^{1} \mathrm{Yi}$-Ping Chang is with Department of Business Mathematics, Soochow University, Taipei, Taiwan
}

[8-10]. In this paper, we mainly discuss the unification of SRGMs based on nonhomogeneous Poisson processes (NHPPs) from a new viewpoint.

The arithmetic, geometric, and harmonic means are three well-known means. Based on them, we consider three more general means - the weighted arithmetic, weighted geometric, and weighted harmonic means [11]. The purpose of this paper is to show how several existing SRGMs based on NHPPs can be derived by applying the concept of weighted arithmetic, weighted geometric, or weighted harmonic mean. Moreover, we propose a general NHPP model from the concept of the quasi-arithmetic mean [11]. Based on this elegant and comprehensive general NHPP model, we can not only derive some existing NHPP models but also can derive some new NHPP models.

In Section 2, several existing SRGMs based on NHPP models are reviewed. In Section 3, the weighted arithmetic, weighted geometric, weighted harmonic, and quasi-arithmetic means are reviewed. The general models of the discrete and the continuous NHP$\mathrm{P}$ models from the viewpoint of the quasi-arithmetic mean are proposed and discussed in Sections 4 and 5, respectively. The conclusions are given in Section 6 .

\section{Nonhomogeneous Poisson Process Models}

In this paper, we will show that several SRGMs based on NHPPs can be treated as special cases of our new general model. While other NHPP models exist, it is our assertion that most SRGMs based on NHPPs can also be treated as special cases of this general model.

Let $\{N(t), t \geq 0\}$ denote a counting process representing the cumulative number of errors detected by time $t$. An SRGMs based on an NHPP with the mean 
value function $m(t)$ can be formulated as [2]

$$
P\{N(t)=n\}=\frac{m(t)^{n}}{n !} e^{-m(t)}, \quad n=0,1,2, \cdots,
$$

where $m(t)$ represents the expected cumulative number of errors detected by time $t$. The mean value function $m(t)$ is nondecreasing in testing time $t$ with the bounded condition $m(\infty)=a$ where $a$ is the expected number of errors to be eventually detected. Generally, we can get different NHPP models by using different nondecreasing mean value functions.

The intensity function at testing time $t$ is [2]

$$
\lambda(t)=m^{\prime}(t)
$$

The software reliability, the probability that no failures occur in $(s, s+t)$ given that the last failure occurred at testing time $s(s \geq 0, t>0)$, is [2]

$$
R(t \mid s)=\exp \{-[m(t+s)-m(s)]\}
$$

The error detection rate per error at testing time $t$ is [12]

$$
d(t)=m^{\prime}(t) /(a-m(t))
$$

The NHPP models with different mean value functions are described in the following.

\section{- Goel-Okumoto model [2]}

The mean value function is

$$
m(t)=a\left(1-e^{-b t}\right), \quad a>0, b>0,
$$

where $a$ is the expected number of errors to be eventually detected and $b$ represents the error detection rate per error.

\section{- Gompertz growth curve model [6]}

The mean value function is

$$
m(t)=a k^{b^{t}}, \quad a>0,0<b<1,0<k<1,
$$

where $a$ is the expected number of errors to be eventually detected.

\section{- Logistic growth curve model [6]}

The mean value function is

$$
m(t)=a \frac{1}{1+k e^{-b t}}, \quad a>0, b>0, k>0,
$$

where $a$ is the expected number of errors to be eventually detected.

\section{- Goel generalized NHPP model [13]}

Goel [13] proposed a simple generalization of the Goel-Okumoto model. The mean value function is

$$
m(t)=a\left(1-e^{-b t^{c}}\right), \quad a>0, b>0, c>0,
$$

where $a$ is the expected number of errors to be eventually detected, and $b$ and $c$ are constants that reflect the quality of testing.

\section{- Delayed S-shaped model [6]}

The mean value function is

$$
m(t)=a\left(1-(1+b t) e^{-b t}\right), a>0, b>0,
$$

where $a$ and $b$ are the expected number of errors to be eventually detected and the error detection rate per error, respectively.

\section{- Inflected S-shaped model [14]}

The mean value function is

$$
m(t)=a \frac{\left(1-e^{-b t}\right)}{\left(1+c e^{-b t}\right)}, a>0, b>0, c>0,
$$

where $a$ is the expected number of errors to be eventually detected while $b$ and $c$ are the error detection rate per error and the inflection factor, respectively.

\section{- Modified Duane model [15]}

The mean value function is

$$
m(t)=a\left\{1-\left(\frac{b}{b+t}\right)^{c}\right\}, \quad a>0, b>0, c>0,
$$

where $a$ is the expected number of errors to be eventually detected.

\section{- Two types of software errors model [16]}

There exist two types of errors: Type 1 (Type 2) errors are easy (difficult) to be detected. The mean value function is

$$
m(t)=a \sum_{i=1}^{2} p_{i}\left(1-e^{-b_{i} t}\right), a>0,0<b_{2}<b_{1}<1, p_{1}+p_{2}=1,
$$

where $a$ is the expected number of errors to be eventually detected, $b_{i}$ is the error detection rate per error of Type $i$ error $(i=1,2)$, and $p_{i}$ is the content proportion of Type $i$ error $(i=1,2)$.

- Weibull-type test-effort function model [17]

Yamada et. al. [17] proposed a software reliability growth model incorporating the amount of test-effort expended during the software testing phase. The mean value function is 
$m(t)=\alpha\left\{1-\exp \left\{-b \int_{0}^{t} \mathrm{w}(s) d s\right\}\right\}=a\left\{1-\exp \left\{-b \alpha\left(1-e^{-\beta t^{\gamma}}\right)\right\}\right.$,

where $a, b, \alpha, \beta, \gamma>0, \alpha$ is the total amount of testeffort required by software testing, $\beta$ is a scale parameter, $\gamma$ is a shape parameter, $b$ is the error detection rate per error, and $\mathrm{w}(t)$ is the test-effort function at time $t$ which is described by the Weibull curve.

\section{Weighted Arithmetic, Weighted Geometric, and Weighted Harmonic means}

In this section, we first review three well-known means, the arithmetic, geometric, and harmonic means. Next, the more general means are considered [11].

Let $x \geq 0$ and $y \geq 0$. The arithmetic mean $z$ of $x$ and $y$ is defined as

$$
z=\frac{1}{2} x+\frac{1}{2} y .
$$

More generally, the weighted arithmetic mean $z$ of $x$ and $y$ with weights $w$ and $1-w$ is defined as

$$
z=w x+(1-w) y, \quad 0<w<1 .
$$

The geometric mean $z$ of $x$ and $y$ is defined as

$$
z=\sqrt{x y} .
$$

That is,

$$
\ln z=\frac{1}{2} \ln x+\frac{1}{2} \ln y .
$$

Similarly, the weighted geometric mean $z$ of $x$ and $y$ with weights $w$ and $1-w$ is defined as

$$
\ln z=w \ln x+(1-w) \ln y, \quad 0<w<1 .
$$

The harmonic mean $z$ of $x$ aznd $y$ is defined as

$$
\frac{1}{z}=\frac{1}{2 x}+\frac{1}{2 y} .
$$

Similarly, the weighted harmonic mean $z$ of $x$ and $y$ with weights $w$ and $1-w$ is defined as

$$
\frac{1}{z}=w \frac{1}{x}+(1-w) \frac{1}{y}, \quad 0<w<1 .
$$

Proposition 1. [11] Let $z_{1}, z_{2}$, and $z_{3}$ are the weighted arithmetic, weighted geometric, and weighted harmonic means of two nonnegative real numbers $x$ and $y$ with weights $w$ and $1-w$, and $0<w<1$. Then,

$$
\min (x, y) \leq z_{3} \leq z_{2} \leq z_{1} \leq \max (x, y)
$$

with equality holding if and only if $x=y$.

A more general mean [11] than the above three ones is considered in the following.

Definition 1. [11] Let $g$ be a real-valued and strictly monotone function. Let $x$ and $y$ are two nonnegative real numbers. The quasi-arithmetic mean $z$ of $x$ and $y$ with weights $w$ and $1-w$ is defined as

$$
z=g^{-1}(w g(x)+(1-w) g(y)), 0<w<1,
$$

where $g^{-1}$ is the inverse function of $g$.

Obviously, from Eq.(8), we can get the weighted arithmetic, weighted geometric, and weighted harmonic means for $g(x)=x, g(x)=\ln x$, and $g(x)=$ $1 / x$, respectively.

Proposition 2. [11] Let $z$ be the quasi-arithmetic mean of two nonnegative real numbers $x$ and $y$ with weights $w$ and $1-w$, and $0<w<1$. Then,

$$
\min (x, y) \leq z \leq \max (x, y)
$$

with equality holding if and only if $x=y$.

\section{A General Discrete Software Reliability Growth Model}

For software reliability growth models, the calendar time or the machine execution time is often used as the unit of software error detection period. However, the appropriate unit of the software error detection period is sometimes the number of test runs [1]. Therefore, Yamada et. al. [18] proposed a general description of a discrete software reliability growth model based on an NHPP, in which the random variable is defined as the number of errors detected by $i$ test runs $(i=$ $0,1,2, \cdots)$.

Let $\{N(i), i=0,1,2 \cdots\}$ denote a discrete counting process representing the cumulative number of errors detected by $i$ test runs. An SRGM based on an NHPP with mean value function $m(i)$ can be formulated as

$$
P\{N(i)=n\}=\frac{m(i)^{n}}{n !} e^{-m(i)}, i, n=0,1,2, \cdots,
$$

where $m(i)$ represents the expected cumulative number of errors detected by $i$ test runs. 
In this section, we propose and discuss a general discrete NHPP model. For the discrete Goel-Okumoto model [18], suppose that the expected number of errors detected per test run is proportional to the current error content of a software system, that is,

$$
m(i+1)-m(i)=b\{a-m(i)\}, a>0,0<b<1,
$$

where $a=m(\infty)$ is the expected number of software errors to be eventually detected and $b$ is the error detection rate per error which is a constant. Taking $w=1-b$, we have

$$
m(i+1)=w m(i)+(1-w) a, \quad 0<w<1, a>0 .
$$

Eq.(12) indicates that $m(i+1)$ is equal to the weighted arithmetic mean of $m(i)$ and $a$ with weights $w$ and $1-w$. That is, the discrete Goel-Okumoto model can also be derived from the viewpoint of the weighted arithmetic mean. Since the weighted arithmetic, weighted geometric, and weighted harmonic means are three well-known means, we thus try to apply the other two means to derived other existing NHPP models. First, consider the case that $m(i+1)$ is equal to the weighted geometric mean of $m(i)$ and $a$ with weights $w$ and $1-w$, then

$$
\frac{1}{m(i+1)}=w \frac{1}{m(i)}+(1-w) \frac{1}{a}, 0<w<1, a>0 .
$$

Next, consider the case that $m(i+1)$ is equal to the weighted harmonic mean of $m(i)$ and $a$ with weights $w$ and $1-w$, then

$$
\ln m(i+1)=w \ln m(i)+(1-w) \ln a, 0<w<1, a>0 .
$$

More generally, let $g$ be a real-valued and strictly monotone function and $m(i+1)$ be equal to the quasiarithmetic mean of $m(i)$ and $a$ with weights $w$ and $1-w$, then

$g(m(i+1))=w g(m(i))+(1-w) g(a), 0<w<1, a>0$.

Solving Eq.(15) yields

$$
\begin{aligned}
g(m(i)) & =w^{i} g(m(0))+\left(1+w+w^{2}+\cdots+w^{i-1}\right)(1-w) g(a) \\
& =w^{i} g(m(0))+\left(1-w^{i}\right) g(a) .
\end{aligned}
$$

Therefore,

$$
m(i)=g^{-1}\left(w^{i} g(m(0))+\left(1-w^{i}\right) g(a)\right),
$$

where $a=m(\infty)$ is the expected number of errors to be eventually detected.
Moreover, by Eq.(15) and Proposition 2, $m(i)$ in Eq.(17) is nondecreasing in $i$, that is, the growth curve of the cumulative number of detected errors is nondecreasing. In the following, we show that three classical SRGMs based on NHPPs can be derived by assigning $g(x)=x, g(x)=1 / x$, and $g(x)=\ln x$ in Eq.(17), respectively.

\section{- Goel-Okumoto model}

Take $g(x)=x$ (i.e., $g^{-1}(x)=x$ ) and $w=e^{-b}$, then

$$
m(i)=w^{i} g(m(0))+\left(1-w^{i}\right) g(a)=a\left(1-k e^{-b i}\right),
$$

where $k=1-m(0) / a, b>0$, and $0<k \leq 1$. If $k=1$ (i.e., $m(0)=0$ ), then

$$
m(i)=a\left(1-e^{-b i}\right), a>0, b>0 .
$$

\section{- Gompertz growth curve}

Take $g(x)=\ln x$ (i.e., $g^{-1}(x)=e^{x}$ ) and $w=b$, then

$$
m(i)=\exp \left\{w^{i} \ln m(0)+\left(1-w^{i}\right) \ln a\right\}=a k^{b^{i}},
$$

where $k=m(0) / a, a>0,0<b<1$, and $0<k<1$.

\section{- Logistic growth curve}

Take $g(x)=1 / x$ (i.e., $\left.g^{-1}(x)=1 / x\right)$ and $w=e^{-b}$, then

$$
m(i)=\frac{1}{w^{i} / m(0)+\left(1-w^{i}\right) / a}=\frac{a}{1+k e^{-b i}},
$$

where $k=a / m(0)-1, b>0$, and $k>0$.

In the following, we consider the more general case that $w$ in Eq.(15) is not a constant for all $i$. That is, let $m(i+1)$ be equal to the quasi-arithmetic mean of $m(i)$ and $a$ with weights $w(i)$ and $1-w(i)$. Eq.(15) can be generalized to be

$$
g(m(i+1))=w(i) g(m(i))+(1-w(i)) g(a) .
$$

Solving Eq.(18) yields

$$
\begin{aligned}
g(m(i)) & =\prod_{j=1}^{i} w(j) g(m(0))+\left(1-\prod_{j=1}^{i} w(j)\right) g(a) \\
& =u_{i} g(m(0))+\left(1-u_{i}\right) g(a),
\end{aligned}
$$

where $u_{0}=1$ and $u_{i}=\prod_{j=1}^{i} w(j)$ for $i \geq 1$. Therefore,

$$
m(i)=g^{-1}\left(u_{i} g(m(0))+\left(1-u_{i}\right) g(a)\right),
$$

is the general discrete NHPP model if $g$ is a realvalued and strictly monotone function. By Eq.(18) and Proposition 2, $m(i)$ in Eq.(20) is nondecreasing in 
i. Obviously, $0<u_{i}<1$ for $i \geq 1$ and $u_{i}$ is decreasing to 0 as $i \rightarrow \infty$. In addition, Eq.(20) can be interpreted that $m(i)$ is equal to the quasi-arithmetic mean of $m(0)$ and $a$ with weights $u_{i}$ and $1-u_{i}$.

For $g(x)=x$ in Eq.(20), we have

$$
\begin{aligned}
m(i) & =u_{i} m(0)+\left(1-u_{i}\right) a=a\left(1-(1-m(0) / a) u_{i}\right) \\
& =a\left(1-k u_{i}\right)
\end{aligned}
$$

where $k=1-m(0) / a$ and $0<k \leq 1$.

For $g(x)=\ln x$ in Eq. $(20)$, we have

$$
m(i)=a\left(\frac{m(0)}{a}\right)^{u_{i}}=a k^{u_{i}}
$$

where $k=m(0) / a$ and $0<k<1$.

For $g(x)=1 / x$ in Eq. $(20)$, we have

$$
m(i)=\frac{a}{1+(a / m(0)-1) u_{i}}=\frac{a}{1+k u_{i}},
$$

where $k=a / m(0)-1$ and $k>1$.

Since the Goel-Okumoto model, the Gompertz growth curve model, and the Logistic growth curve model have been discussed in the above subsection, we will show that some other SRGM based on NHPPs can also be directly derived from Eq.(21) in the following.

\section{- Goel generalized NHPP model}

Take $g(x)=x$ and $u_{i}=e^{-b i^{c}}$ (i.e., $w(i)=$ $\left.e^{-b\left\{i^{c}-(i-1)^{c}\right\}}\right)$, and then

$m(i)=a\left\{1-k e^{-b i^{c}}\right\}, \quad a>0, b>0, c>0,0<k \leq 1$.

Furthermore, if $k=1$ (i.e., $m(0)=0$ ), we have

$m(i)=a\left\{1-e^{-b i^{e}}\right\}, \quad a>0, b>0, c>0,0<k \leq 1$.

In this model, $w(i)$ is increasing in $i$ if $c<1, w(i)$ is a constant if $c=1$, and $w(i)$ is decreasing in $i$ if $c>1$.

\section{- Delayed S-shaped model}

Take $g(x)=x$ and $u_{i}=(1+b i) e^{-b i}$ (i.e., $w(i)=$ $\left.\left\{\frac{1+b i}{1+b(i-1)}\right\} e^{-b}\right)$, and then

$m(i)=a\left\{1-k(1+b i) e^{-b i}\right\}, \quad a>0, b>0,0<k \leq 1$.

Furthermore, if $k=1$ (i.e., $m(0)=0$ ), we have

$$
m(i)=a\left\{1-(1+b i) e^{-b i}\right\}, \quad a>0, b>0 .
$$

In this model, $w(i)$ is decreasing in $i$.

\section{- Inflected S-shaped model}

Take $g(x)=x$ and $u_{i}=\frac{(1+c) e^{-b i}}{1+c e^{-b i}}$ (i.e., $w(i)=$ $\left.\left\{\frac{1+c e^{-b(i-1)}}{1+c e^{-b i}}\right\} e^{-b}\right)$, and then $m(i)=a\left\{1-k \frac{(1+c) e^{-b i}}{1+b e^{-b i}}\right\}, a>0, b>0, c>0,0<k \leq 1$.

Furthermore, if $k=1$ (i.e., $m(0)=0$ ), we have

$$
m(i)=a \frac{1-e^{-b i}}{1+c e^{-b i}}, \quad a>0, b>0, c>0 .
$$

In this model, $w(i)$ is decreasing in $i$.

\section{A General Continuous Software Reliability Growth Model}

In this section, we propose and discuss a general continuous NHPP model. As the above discussion in the discrete case, let $m(t+\Delta t)$ be equal to the quasiarithmetic mean of $m(t)$ and $a$ with weights $w(t, \Delta t)$ and $1-w(t, \Delta t)$, and then

$$
g(m(t+\Delta t))=w(t, \Delta t) g(m(t))+(1-w(t, \Delta t)) g(a),
$$

where $0<w(t, \Delta t)<1$ and $g$ is a real-valued, strictly monotone, and differentiable function. That is,

$\frac{g(m(t+\Delta t))-g(m(t))}{\Delta t}=\frac{1-w(t, \Delta t)}{\Delta t}(g(a)-g(m(t)))$.

Suppose $(1-w(t, \Delta t)) / \Delta t \rightarrow b(t)$ as $\Delta t \rightarrow 0$, we get the differential equation

$$
\frac{\partial}{\partial t} g(m(t))=b(t)\{g(a)-g(m(t))\}
$$

For $g(x)=x$ in Eq.(25) (i.e., the weighted arithmetic mean considered), we have

$$
\frac{\partial}{\partial t} m(t)=b(t)(a-m(t))
$$

Here, $b(t)$ is the error detection rate per error [18]. Furthermore, if $b(t)=b$, then the Goel-Okumoto model can be derived from Eq.(26). The differential equations for $g(x)=\ln x$ and $g(x)=1 / x$ can also be derived from Eq.(25), respectively.

Theorem 1. Let $\partial g(m(t)) / \partial t=b(t)\{g(a)-g(m(t))\}$, where $g$ is a real-valued, strictly monotone, and differentiable function. We have

$$
m(t)=g^{-1}\left(g(a)+[g(m(0))-g(a)] e^{-B(t)}\right),
$$


where

$$
B(t)=\int_{0}^{t} b(u) d u
$$

Proof: Product $e^{B(t)}$ to both sides of Eq.(25), then

$$
e^{B(t)} \frac{\partial}{\partial t} g(m(t))+e^{B(t)} b(t) g(m(t))=e^{B(t)} b(t) g(a) .
$$

Therefore,

$$
\frac{\partial}{\partial t}\left(e^{B(t)} g(m(t))\right)=\frac{\partial}{\partial t}\left(g(a) e^{B(t)}\right) .
$$

Hence,

$$
g(m(t))=g(a)+c e^{-B(t)} .
$$

Since $B(0)=0$, then $c=g(m(0))-g(a)$, (i) of Theorem 1 can be proved.

Note that $m(t)$ in Eq.(27) is the general model of the continuous NHPP models if $g$ is a real-valued, strictly monotone, and differentiable function. In addition, by Eq.(24) and Proposition 2, $m(t)$ is nondecreasing in $t$. The intensity function, the software reliability, and the error detection rate per error of this general NHPP model are

$$
\begin{aligned}
\lambda(t)=- & \left\{g^{\prime}(m(t))\right\}^{-1}[g(m(0))-g(a)] b(t) e^{-B(t)}, \\
R(t \mid s)= & \exp \left\{g^{-1}\left(g(a)+[g(m(0))-g(a)] e^{-B(s)}\right)\right. \\
& \left.-g^{-1}\left(g(a)+[g(m(0))-g(a)] e^{-B(t+s)}\right)\right\} \\
d(t)= & \frac{b(t)}{a-m(t)} \frac{g(a)-g(m(t))}{g^{\prime}(m(t))} .
\end{aligned}
$$

The values of $m(t), \lambda(t), R(t \mid s)$, and $d(t)$ for $g(x)=x$, $g(x)=1 / x$, and $g(x)=\ln x$ can be easily obtained in the following three Corollaries, respectively.

Corollary 1. From the viewpoint of the weighted arithmetic mean, take $g(x)=x$ in Eq.(27) and let $k=1-m(0) / a$, then

(i) $m(t)=a\left(1-k e^{-B(t)}\right), \quad a>0,0<k \leq 1$;

(ii) $\lambda(t)=a k b(t) e^{-B(t)}$;

(iii) $R(t \mid s)=\exp \left\{-a k\left(e^{-B(s)}-e^{-B(t+s)}\right)\right\}$;

(iv) $d(t)=b(t)$.

Proof: This Corollary can be easily proved.

Corollary 2. From the viewpoint of the weighted geometric mean, take $g(x)=\ln x$ in Eq.(27) and let $k=m(0) / a$, then (i) $m(t)=a k^{e^{-B(t)}} a>0,0<k<1$;

(ii) $\lambda(t)=-a(\ln k) b(t) e^{-B(t)} k^{e^{-B(t)}}$;

(iii) $R(t \mid s)=\exp \left\{-a\left[k^{e^{-B(t+s)}}-k^{e^{-B(s)}}\right]\right\}$;

(iv) $d(t)=b(t) m(t)(\ln a-\ln m(t)) /(a-m(t))$;

(v) if $b(t)$ is nondecreasing in $t$, then $d(t)$ is nondecreasing in $t$.

Proof: To prove (v), let

$$
h(t)=\frac{m(t)(\ln a-\ln m(t))}{a-m(t)}
$$

In the following, we want to show that $h(t)$ is nondecreasing in $t$.

$h^{\prime}(t)=\frac{m^{\prime}(t)\{(\ln a-\ln m(t)-1)(a-m(t)+m(t)(\ln a-\ln m(t))\}}{[a-m(t)]^{2}}$.

The numerator of Eq.(32) is equal to

$$
m^{\prime}(t)\{m(t)+a(\ln a-\ln m(t)-1)\} .
$$

Let

$$
f(t)=m(t)+a(\ln a-\ln m(t)-1) .
$$

By a simple calculation, it can be shown that $f^{\prime}(t) \leq 0$ and $\lim _{t \rightarrow \infty} f(t)=0$. Hence, we have $f(t) \geq 0$ for al$1 t$. Since $m^{\prime}(t) \geq 0$ and $[a-m(t)]^{2}>0$, we have $h^{\prime}(t) \geq 0$, that is, $h(t)$ is nondecreasing. Thus, (v) of Corollary 2 can be proved.

Corollary 3. From the viewpoint of the weighted harmonic mean, take $g(x)=1 / x$ in Eq.(27) and let $k=a / m(0)-1$, then

(i) $m(t)=a \frac{1}{1+k e^{-B(t)}}, \quad a>0, k>0$;

(ii) $\lambda(t)=\frac{a k b(t)}{\left\{1+k e^{-B(t)}\right\}^{2}} e^{-B(t)}$;

(iii) $R(t \mid s)=\exp \left\{-a\left\{\frac{1}{1+k e^{-B(t+s)}}-\frac{1}{1+k e^{-B(s)}}\right\}\right\}$;

(iv) $d(t)=b(t) m(t) / a$;

(v) if $b(t)$ is nondecreasing in $t$, then $d(t)$ is nondecreasing in $t$.

Proof: This Corollary can be easily proved.

Proposition 3. Let $m_{A}(t), m_{G}(t)$, and $m_{H}(t)$ are three mean value functions defined by Eqs.(26), (27), 
and (28), respectively. Moreover, suppose the values of $a, m(0)$, and $b(t)$ in the above three mean value functions are all equal. We have:

$$
m_{A}(t)>m_{G}(t)>m_{H}(t) .
$$

Proof: It can be easily proved by Proposition 1.

In the following, we show that some classical $\mathrm{S}$ RGMs based on NHPPs can be directly derived from Corollary 1, 2, or 3 .

\section{- Goel-Okumoto model}

Take $g(x)=x$ and $b(t)=b$, then from Corollary 1 , $m(t)=a\left(1-k e^{-b t}\right), \quad a>0, b>0,0<k \leq 1$.

If $k=1$ (i.e., $m(0)=0$ ), then

$$
m(t)=a\left(1-e^{-b t}\right), \quad a>0, b>0 .
$$

In this model, $b(t)$ is a constant.

\section{- Gompertz growth curve}

Take $g(x)=\ln x$ and $b(t)=b$, then from Corollary 2,

$$
m(t)=a k^{e^{-b t}}, \quad a>0, b>0,0<k<1 .
$$

In this model, b(t) is a constant.

\section{- Logistic growth curve}

Take $g(x)=1 / x$ and $b(t)=b$, then from Corollary 3 ,

$$
m(t)=a \frac{1}{1+k e^{-b t}}, \quad a>0, b>0, k>0 .
$$

In this model, $b(t)$ is a constant.

\section{- Goel Generalized NHPP Model}

Take $g(x)=x$ and $b(t)=b c t^{c-1}$, then from Corollary 1 ,

$m(t)=a\left(1-k e^{-b t^{c}}\right), \quad a>0, b>0, c>0,0<k \leq 1$.

If $k=1$ (i.e., $m(0)=0$ ), then

$$
m(t)=a\left(1-e^{-b t^{c}}\right), \quad a>0, b>0, c>0 .
$$

In this model, $b(t)$ is increasing if $c>1, b(t)$ is a constant if $c=1$, and $b(t)$ is decreasing if $c<1$.

\section{- Delayed S-shaped model}

Take $g(x)=x$ and $b(t)=b^{2} t /(1+b t)$, then from Corollary 1,

$m(t)=a\left\{1-k(1+b t) e^{-b t}\right\}, \quad a>0, b>0,0<k \leq 1$.
If $k=1$ (i.e., $m(0)=0$ ), then

$$
m(t)=a\left\{1-(1+b t) e^{-b t}\right\}, \quad a>0, b>0 .
$$

In this model, $b(t)$ is increasing in $t$.

\section{- Inflected S-shaped model}

Take $g(x)=x$ and $b(t)=b /\left(1+c e^{-b t}\right)$, then from Corollary 1,

$m(t)=a\left\{1-k \frac{(1+c) e^{-b t}}{1+c e^{-b t}}\right\}, a>0, b>0, c>0,0<k \leq 1$.

If $k=1$ (i.e., $m(0)=0$ ), then

$$
m(t)=a \frac{\left(1-e^{-b t}\right)}{\left(1+c e^{-b t}\right)}, a>0, b>0, c>0 .
$$

In this model, $b(t)$ is increasing in $t$.

\section{- Modified Duane model}

Take $g(x)=x$ and $b(t)=c /(b+t)$, then from Corollary 1,

$m(t)=a\left\{1-k\left(\frac{b}{b+t}\right)^{c}\right\}, a>0, b>0, c>0,0<k \leq 1$.

If $k=1$ (i.e., $m(0)=0$ ), then

$$
m(t)=a\left\{1-\left(\frac{b}{b+t}\right)^{c}\right\}, \quad a>0, b>0, c>0 .
$$

In this model, $b(t)$ is decreasing in $t$.

\section{- Two types of software errors model}

Take $g(x)=x$ and $b(t)=\left(\sum_{i=1}^{2} p_{i} b_{i} e^{-b_{i} t}\right) /\left(\sum_{i=1}^{2} p_{i} e^{-b_{i} t}\right)$, then from Corollary 1 ,

$$
m(t)=a \sum_{i=1}^{2} p_{i}\left(1-k e^{-b_{i} t}\right), 0<k \leq 1 .
$$

If $k=1$ (i.e., $m(0)=0$ ), then

$$
m(t)=a \sum_{i=1}^{2} p_{i}\left(1-e^{-b_{i} t}\right),
$$

where $a>0,0<b_{2}<b_{1}<1, p_{1}+p_{2}=1,0<p_{1}<0,0<$ $p_{2}<1$. In this model, $b(t)$ is decreasing in $t$ [16].

\section{- Weibull-type testing-effort function model}

Take $g(x)=x$ and $b(t)=b \alpha \beta \gamma t^{\gamma-1} e^{-\beta t^{\gamma}}$, then from Corollary 1 ,

$$
m(t)=a\left\{1-k \exp \left\{-b \alpha\left(1-e^{-\beta t^{\gamma}}\right)\right\}\right\}, 0<k \leq 1 .
$$

Furthermore, if $k=1$ (i.e., $m(0)=0$ ), then $m(t)=a\left\{1-\exp \left\{-b \alpha\left(1-e^{-\beta t^{\gamma}}\right)\right\}\right\}, a, b, \alpha, \beta, \gamma>0$. 


\section{Conclusions}

In this paper, we first show how several existing software reliability growth models based on NHPPs can be derived by applying the concept of the weighted arithmetic, weighted geometric, or weighted harmonic mean. Next, we proposed a general NHPP model from the viewpoint of quasi-arithmetic mean, a more general mean of the above three weighted means. Many existing SRGMs based on NHPPs can be derived as special cases of this general NHPP model. Furthermore, while other NHPP models exist, it is our assertion that most SRGMs based on NHPPs can also be treated as special cases of this general model.

Acknowledgment. We would like to express our gratitude for the support of the National Science Council, Taiwan, under Grants NSC86-2213-E002040. Reviewers' comments are also highly appreciated.

\section{References}

[1] C. V. Ramamoorthy and F. B. Bastani, "Software reliability - Status and perspectives", IEEE Trans. Software Engineering, Vol. 8, No. 4, pp. 354-371, 1982.

[2] A. L. Goel and K. Okumoto, "Time-dependent error-detection rate model for software reliability and other performance measures", IEEE Trans. Reliability, Vol. 28, No.3, pp. 206-211, 1979.

[3] B. Littlewood and J. L. Verrall, "A Bayesian reliability growth model for computer software", Journal Royal Statistical Society-Series $C$, Vol. 22, No.3, pp. 332-346, 1973.

[4] Z. Jelinski and P. B. Moranda, "Software reliability research", in Statistical Computer Performance Evaluation, W. Freiberger, Editor, Academic, New York, pp. 465-484, 1972.

[5] J. D. Musa and K. Okumoto, "A logarithmic Poisson execution time model for software reliability measurement", Proc. 7th Int. Conf. Software Engineering, pp. 230-238, 1984.

[6] S. Yamada, M. Ohba, and S. Osaki, "S-shaped reliability growth modeling for software error detection", IEEE Trans. Reliability, Vol. 32, No. 5, pp. $475-478,1983$.
[7] J. D. Musa, A. Iannino, and K. Okumoto, Software Reliability - Measurement, Prediction, Application, McGraw-Hill, INc., 1987.

[8] N. Langberg and N. D. Singpurwalla, "A unification of some software reliability models", SIAM J. Sci. Stat. Comput., Vol. 6, No.3, pp. 781-790, 1985 .

[9] D. R. Miller, "Exponential order statistic models of software reliability growth", IEEE Trans. Software Engineering, Vol. 12, No.1, pp. 12-24, 1986.

[10] M. Trachtenberg, "A general theory of softwarereliability modeling", IEEE Trans. Reliability, Vol. 39, No. 1, pp. 92-96, 1990.

[11] P. S. Bullen, D. S. Mitrinović, and P. M. Vasić, Means and Their Inequalities, D. Reidel Publishing Company, Dordrecht, Holland, 1988.

[12] S. Yamada and S. Osaki, "Software reliability growth modeling: models and applications", IEEE Trans. Software Engineering, Vol. 11, No. 12, pp. 1431-1437, 1985.

[13] A. L. Goel, "Software reliability models: assumptions, limitations, and applicability", IEEE Trans. Software Engineering, Vol. 11, No. 12, pp. 1411-1423, 1985.

[14] M. Ohba, "Software Reliability Analysis Models", IBM J. Research and Development, Vol. 28, No. 4, pp. 428-443, 1984

[15] B. Littlewood, "Rationale for a modified Duane model", IEEE Trans. Reliability, Vol. 33, pp. $157-159,1984$.

[16] S. Yamada and S. Osaki, "Nonhomogeneous error detection rate models for software reliability growth", in Stochastic Models in Reliability Theory, S. Osaki and Y. Hatoyama, Eds., Berlin: Springer-Verlag, pp. 120-143, 1984.

[17] S. Yamada, J. Hishitani, and S. Osaki, "Softwarereliability growth with a weibull test-effort: a model \& application", IEEE Trans. Reliability, Vol. 42, No. 1, pp. 100-106, 1993.

[18] S. Yamada and S. Osaki, "Discrete software reliability growth models", Applied Statisitic Models and Data Analysis, Vol. 1, pp. 65-77, 1985. 